\title{
DECAIMENTO DE ÍONS CIANATO E A FORMAÇÃO DE CARBAMATO DE ETILA
}

\author{
Alexandre Ataide da Silva, Thiago Hideyuki Kobe Ohe e Douglas Wagner Franco* \\ Instituto de Química de São Carlos, Universidade de São Paulo, CP 780, 13560-970 São Carlos - SP, Brasil
}

Recebido em 12/11/12; aceito em 3/5/13; publicado na web em 1/7/13

\begin{abstract}
CYANATE ION DECAY AND ETHYL CARBAMATE FORMATION. Cyanate ion stability was studied in aqueous/ethanolic solutions, $\mathrm{pH}=4.5\left(\mathrm{CH}_{3} \mathrm{COOH} / \mathrm{NaCH}_{3} \mathrm{COO}\right)$, at different temperatures. Following the decay $\left[(\mathrm{OCN})^{-}\right]$versus time, in the presence of excess $\mathrm{C}_{2} \mathrm{H}_{5} \mathrm{OH}$, the rate constant for this reaction $\left(k_{1}\right)$ was calculated as $(2.5 \pm 0.3) \times 10^{-4} \mathrm{~s}^{-1}$ at $25{ }^{\circ} \mathrm{C}$, for $0 \leq\left[\mathrm{C}_{2} \mathrm{H}_{5} \mathrm{OH}\right] \leq$ $13.7 \times 10^{-1} \mathrm{~mol} \mathrm{~L}^{-1}$. For $\left[\mathrm{C}_{2} \mathrm{H}_{5} \mathrm{OH}\right] \geq 2.0 \mathrm{~mol} \mathrm{~L}^{-1}$ a decrease in the numerical value of $k_{1}$ was observed, reaching $5.2 \times 10^{-5} \mathrm{~s}^{-1}$ when $\left[\mathrm{CH}_{3} \mathrm{CH}_{2} \mathrm{OH}\right]=13.7 \mathrm{~mol} \mathrm{~L}^{-1}$. Variations in the kinetic parameter values $\Delta H_{1}^{*}, \Delta S_{1}^{*}$ and $\Delta G_{I^{*}}^{*}$ for the cyanate ion decay reaction were observed for solutions at different ethanol concentrations.
\end{abstract}

Keywords: cyanate ion; ethyl carbamate formation; aqueous/ethanol solution.

\section{INTRODUÇÃO}

O carbamato de etila $\left(\mathrm{NH}_{2} \mathrm{COOCH}_{2} \mathrm{CH}_{3}\right.$, ocorre naturalmente em alimentos fermentados (pão, iogurte), bebidas alcoólicas fermentadas (vinho, cerveja) e bebidas destiladas (uísque, aguardentes). ${ }^{1-4}$ São diversos os compostos presentes em alimentos e bebidas que na presença de etanol podem levar à formação de carbamato de etila (CE) durante as etapas de processamento e de armazenamento (Esquema 1).

Uma das rotas propostas para a formação do carbamato de etila envolve a reação de decomposição de uréia $\left(\mathrm{H}_{2} \mathrm{NCONH}_{2}\right)$ em meio ácido entre $60^{\circ} \mathrm{C}$ e $100{ }^{\circ} \mathrm{C}$, na presença de etanol (Esquema 1, reação I). Em presença de água a uréia decompõe-se termicamente em amônia $\left(\mathrm{NH}_{3}\right)$, dióxido de carbono $\left(\mathrm{CO}_{2}\right)$, ácidos ciânico $(\mathrm{HOCN})$ e isociânico (HNCO). O ácido ciânico converte-se em ácido isociânico ${ }^{5-11}$ e este último reagiria com o etanol levando à formação de CE (Esquema 1, reação II e Esquema 2). Procurando contribuir para o conhecimento da formação de $\mathrm{CE}$ em aguardentes, descreve-se o decaimento da concentração do íon cianato $(\mathrm{OCN})^{-}$em soluções aquo-etanólicas.
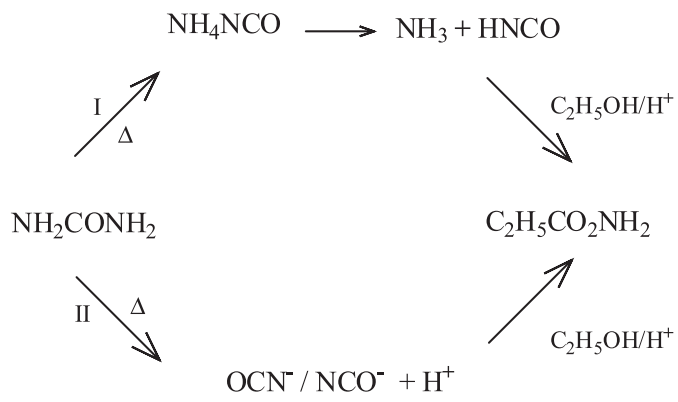

Esquema 1. Formação do carbamato de etila a partir da uréia ${ }^{5-11}$

\section{PARTE EXPERIMENTAL}

\section{Reagentes}

Os reagentes e solventes utilizados, sempre de grau analítico ou cromatográfico, foram adquiridos das seguintes companhias: Sigma-Aldrich (Steinheim, Germany), Mallinckrodt Baker (Paris, United States of America), Fluka (Buchs, Switzerland), Carlo Erba (Milano, Italy) e Tedia (Phillipsburg, United States of America). A

*e-mail: douglas@iqsc.usp.br água utilizada foi previamente destilada e a seguir deionizada por um sistema Milli- $Q$ da Millipore (Bedford, United States of America).

As soluções aquo/etanólicas foram tamponadas a $\mathrm{pH}=4,5$, valor este que corresponde ao valor mediano de $\mathrm{pH}$ apresentado pelas aguardentes, utilizando o sistema tampão ácido acético e acetato de sódio. Estas soluções foram fortificadas com alíquotas de solução estoque de cianato de potássio, recém-preparada. As soluções resultantes foram transferidas para frascos de vidro (vials) de $28 \mathrm{~mL}$, lacrados com septos de borracha e alumínio e então acondicionados em banho termostático. Alíquotas destas soluções foram retiradas de tempos em tempos para o acompanhamento do decaimento do íon cianato.

\section{Decaimento do íon cianato $(\mathrm{OCN})^{-}$}

Foram preparadas soluções nas seguintes concentrações $\left(\mathrm{mol} \mathrm{L}^{-1}\right)$ de etanol: $1,0 \times 10^{-2}, 2,0 \times 10^{-2}, 4,0 \times 10^{-2}, 8,0 \times 10^{-2}, 2,4,8,13,7$. A concentração do íon cianato $(\mathrm{KOCN})$ escolhida experimentalmente foi constante e igual a $1,0 \times 10^{-3} \mathrm{~mol} \mathrm{~L}^{-1}$. A partir destas soluções, acompanhou-se o decaimento da concentração do íon $(\mathrm{OCN})^{-}$em função do tempo, utilizando-se a metodologia baseada na reação de ciclização do ácido 2-aminobenzóico. ${ }^{12}$

Alíquotas de 3,0 mL das soluções aquo/etanólicas contendo KOCN foram misturadas com 3,0 mL de uma solução $0,010 \mathrm{~mol} \mathrm{~L}^{-1}$ de ácido 2-aminobenzóico. A solução obtida foi acondicionada em frascos de vidro (envoltos com papel alumínio) e aquecidas a $37 \pm$ $0,5^{\circ} \mathrm{C}$ em banho seco por 10 minutos. Posteriormente, adicionou-se a esta solução aproximadamente $6,0 \mathrm{~mL}$ de uma solução de ácido clorídrico $\left(4,1 \mathrm{~mol} \mathrm{~L}^{-1}\right)$ seguindo-se seu aquecimento a $65 \pm 0,5{ }^{\circ} \mathrm{C}$ (banho seco) por 10 minutos. A benzoiluréia assim obtida foi resfriada à temperatura ambiente e a absorbância da solução determinada a 310 $\mathrm{nm}\left(\varepsilon=412 \mathrm{~L} \mathrm{~mol}^{-1} \mathrm{~cm}^{-1}\right)^{6}$ utilizando espectrofotômetro UV-visível Hitachi modelo U-3501(Tokio, Japan). A solução de ácido 2-aminobenzóico foi sempre preparada no mesmo dia do seu uso e antes da realização dos experimentos, sendo sempre mantida ao abrigo da luz.

\section{Formação de carbamato de etila}

A formação de carbamato de etila foi acompanhada utilizando-se cromatógrafo para fase gasosa Shimadzu GC-17A acoplado a espectrômetro de massas GCMS-QP5050 operando no modo SIM (monitoramento de íon seletivo com razão $\mathrm{m} / \mathrm{z}=62$ ) usando impacto eletrônico de $70 \mathrm{eV}$. Foi dada preferência ao monitoramento 
do íon $\mathrm{m} / \mathrm{z}=62\left(\left[\mathrm{M}-\mathrm{C}_{2} \mathrm{H}_{3}\right]\right)$ com respeito ao íon $\mathrm{m} / \mathrm{z}=44$, apesar deste último sinal ser o mais abundante nos espectros de massas de substancias orgânicas, quando se utiliza ionização por impacto eletrônico. Assim, este sinal estaria, em tese, mais suscetível a interferências. ${ }^{13,14}$ As amostras foram quantificadas por meio de adição de padrão, utilizando como padrão interno carbamato de propila. Os limites de detecção (LD) e quantificação (LQ) para o CE foram de 10 e $30 \mu \mathrm{g} \mathrm{L}^{-1}$, respectivamente. A separação cromatográfica foi realizada em uma coluna capilar de fase polar, HP-FFAP, com filme de polietilenoglicol esterificado, seguindo metodologia descrita na literarura. ${ }^{15,16}$

\section{Experimentos cinéticos}

As constantes $k_{1}$ foram calculadas por meio de gráficos $\ln \left(\left[(\mathrm{OCN})^{-}\right]_{\infty}-\left[(\mathrm{OCN})^{-}\right]_{t}\right)$ versus tempo e $\ln \left([\mathrm{CE}]_{\infty}-[\mathrm{CE}]_{t}\right)$ versus tempo, respectivamente, onde $\left[(\mathrm{OCN})^{-}\right]$e $[\mathrm{CE}]$ referem-se às concentrações do íon cianato e do carbamato de etila, respectivamente. Os símbolos $\infty$ e t se referem respectivamente ao final da reação e ao tempo em que a medida se realizou. Segundo cálculos utilizando método INDO (Intermediate Neglect of Differential Overlap) a distribuição da carga é igual sobre os átomos de oxigênio e nitrogênio ${ }^{17}$ no íon $(\mathrm{OCN})^{-}$, notação esta que é utilizada no presente trabalho para expressar a soma das concentrações dos íons cianato e isocianato. ${ }^{17,18}$ Considerou-se que a concentração $\left[(\mathrm{OCN})^{-}\right]_{t}$ quando $t=0$ é igual à concentração analítica de cianato de potássio (KOCN) utilizada. Como a metodologia analítica, que permite determinar a variação da concentração do pseudo-haleto ao longo do tempo (t), não faz distinção entre cianato e isocianato e estes estão em rápido equilíbrio, os resultados são interpretados como a soma destes dois ânions. Em se tratando dos ácidos correspondentes: HNCO (isociânico) e HOCN (ciânico), as duas formas existem em equilíbrio com a predominância do primeiro. ${ }^{17,18}$ Cálculos quânticos (DFT- Density Functional Theory) indicam que a reação de formação de carbamato de etila a partir de etanol e ácido isociânico é favorecida com respeito à reação com ácido ciânico. ${ }^{6}$ As reações foram estudadas sempre em condições de pseudo-primeira ordem (excesso de etanol).

Os parâmetros de ativação $\left(\Delta H_{1}^{*}, \Delta S_{1}^{*}\right.$ e $\left.\Delta G_{1}^{*}\right)$ para a reação:

$$
\mathrm{HNCO}+\mathrm{CH}_{3} \mathrm{CH}_{2} \mathrm{OH} \stackrel{k 1}{\rightarrow} \mathrm{NH}_{2} \mathrm{COOCH}_{2} \mathrm{CH}_{3}
$$

Esquema 2. Formação de carbamato de etila a partir do ácido isociânico e etanol

foram calculados, a partir dos valores de $k_{1}$, para a mesma concentração de KOCN e/ou etanol, a diferentes temperaturas, utilizando as Equações de 1-4. ${ }^{19} \mathrm{O}$ valor da energia de ativação $\left(\mathrm{E}_{\mathrm{a}}\right)$ foi calculado graficamente lançando $\log k_{1}$ versus $1 / \mathrm{T}$ (equação 1). A Entropia de ativação $\left(\Delta S_{I^{*}}\right)$ foi calculada para a temperatura de $25^{\circ} \mathrm{C}(298 \mathrm{~K})$, inserindo-se $k_{1}=2,3 \times 10^{-4} \mathrm{~s}^{-1}$, na equação (2) calculado a partir da equação (3) e utilizando a equação (4).

$$
\begin{gathered}
\log k_{1}=\log A-\frac{\mathrm{E}_{\mathrm{a}}}{2,303 \mathrm{RT}} \\
\frac{\Delta S_{1}^{\ddagger}}{2,303 R}=\log k_{1}-\log \left(\frac{e \boldsymbol{k}}{\mathrm{h}}\right)-\log \mathrm{T}+\frac{\mathrm{E}_{\mathrm{a}}}{2,303 R T} \\
\mathrm{E}_{\mathrm{a}}=\Delta H_{l^{\ddagger}}+\mathrm{RT} \\
\Delta G_{I}^{\ddagger}=\Delta H_{I}^{\ddagger}+T \Delta S_{I^{\ddagger}}
\end{gathered}
$$

Nestas equações $A$ é a constante pré-exponencial, $\boldsymbol{k}$ a constante de Boltzmann $\left(1,381 \times 10^{-23} \mathrm{~J} \mathrm{~K}^{-1}\right), h$ a constante de Planck $(6,626 \mathrm{x}$ $10^{-34} \mathrm{~J} \mathrm{~s}$ ), T é a temperatura (Kelvin, $K$ ), $\Delta H_{I}^{\ddagger}$ variação de entalpia de ativação, $\Delta S_{I}^{*}$ é a variação de entropia de ativação, $\Delta G_{I}^{*}$ é a energia livre de ativação de Gibbs e $R$ é a constante universal dos gases $\left(1,987 \mathrm{cal} \mathrm{K}^{-1} \mathrm{~mol}^{-1}\right){ }^{5,6}$

\section{RESULTADOS E DISCUSSÃO}

O perfil típico das curvas absorbância versus tempo, para o decaimento do íon cianato, bem como para os gráficos $\ln \left(\left[(\mathrm{OCN})^{-}\right]_{\infty}-\left[(\mathrm{OCN})^{-}\right]_{t}\right)$ versus tempo, estão ilustrados na Figura 1 e os dados experimentais reunidos na Tabela 1. Estes resultados indicam que a constante de velocidade para esta reação $\left(k_{l}=2,3 \pm 0,3 \times 10^{-4} \mathrm{~s}^{-1}\right)$, não varia dentro do erro experimental no intervalo de concentração de etanol de 0 a $8,0 \times 10^{-1} \mathrm{~mol} \mathrm{~L}^{-1}$.

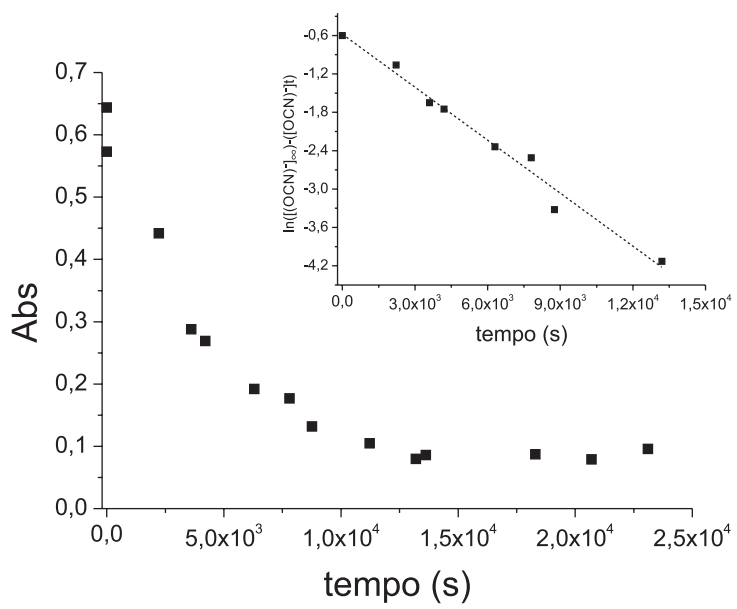

Figura 1. Decaimento do íon cianato em função do tempo e gráfico do $\ln \left(\left[(\mathrm{OCN})^{-}\right]_{\infty}-\left[(\mathrm{OCN})^{-}\right]_{t}\right)$ versus tempo (insert). $\left(\mathrm{T}=25^{\circ} \mathrm{C}(298 \mathrm{~K})\right.$, $\left.\left[\mathrm{CH}_{3} \mathrm{CH}_{2} \mathrm{OH}\right]=2,0 \times 10^{-2} \mathrm{~mol} \mathrm{~L} L^{-1}\right)$

É interessante ressaltar que em meio aquoso e na ausência de etanol, a concentração de (OCN) decai com $k_{1}=2,9 \times 10^{-4} \mathrm{~s}^{-1}$, (Tabela 1) e que nestas condições não ocorre a formação de carbamato de etila.

Tabela 1. Valores das constantes de velocidades obtidas pelo gráfico de $\ln$ $\left(\left[\mathrm{OCN}^{-}\right]_{\infty}-\left[\mathrm{OCN}^{-}\right]_{1}\right)$ versus tempo em diferentes $\left[\mathrm{CH}_{3} \mathrm{CH}_{2} \mathrm{OH}\right]$

\begin{tabular}{cccc}
\hline $\begin{array}{c}\text { Concentração EtOH } \\
(\mathrm{mol} \mathrm{L})\end{array}$ & $k_{1}, \mathrm{~s}^{-1}$ & $\mathrm{t}_{1 / 2}, \mathrm{~s}^{-1}$ & $\mathrm{~T},{ }^{\circ} \mathrm{C}$ \\
\hline 0 & $2,9 \times 10^{-4}$ & $2,4 \times 10^{-3}$ & 25 \\
$1,0 \times 10^{-2}$ & $3,3 \times 10^{-4}$ & $2,1 \times 10^{-3}$ & 25 \\
$2,0 \times 10^{-2}$ & $2,3 \times 10^{-4}$ & $3,0 \times 10^{-3}$ & 25 \\
$2,0 \times 10^{-2}$ & $4,5 \times 10^{-4}$ & $1,5 \times 10^{-3}$ & 35 \\
$2,0 \times 10^{-2}$ & $9,2 \times 10^{-4}$ & $7,5 \times 10^{-2}$ & 45 \\
$4,0 \times 10^{-2}$ & $2,3 \times 10^{-4}$ & $2,9 \times 10^{-3}$ & 25 \\
$8,0 \times 10^{-2}$ & $2,4 \times 10^{-4}$ & $2,3 \times 10^{-3}$ & 25 \\
$8,0 \times 10^{-1}$ & $2,5 \times 10^{-4}$ & $2,8 \times 10^{-3}$ & 25 \\
2,0 & $1,7 \times 10^{-4}$ & $4,1 \times 10^{-3}$ & 25 \\
4,0 & $1,5 \times 10^{-4}$ & $4,6 \times 10^{-3}$ & 25 \\
6,8 & $6,7 \times 10^{-5}$ & $1,0 \times 10^{-4}$ & 25 \\
6,8 & $2,0 \times 10^{-4}$ & $3,4 \times 10^{-3}$ & 35 \\
6,8 & $5,5 \times 10^{-4}$ & $1,2 \times 10^{-3}$ & 45 \\
8,0 & $6,8 \times 10^{-5}$ & $1,1 \times 10^{-4}$ & 25 \\
13,7 & $5,2 \times 10^{-5}$ & $1,3 \times 10^{-4}$ & 25 \\
\hline
\end{tabular}


Os valores de $k_{1}$ para o decaimento de íons (OCN)- em soluções aquosas na ausência de etanol, são concordantes dentro do erro experimental com os valores calculados para soluções aquo-etanólicas de mesma concentração hidrogeniônica e concentrações de etanol na faixa de $1,0 \times 10^{-2}$ a $8,0 \times 10^{-1} \mathrm{~mol} \mathrm{~L}^{-1}$.

A reação de formação de carbamato de etila foi acompanhada nas mesmas condições experimentais $\left(\left[\mathrm{CH}_{3} \mathrm{CH}_{2} \mathrm{OH}\right]=8,0 \times 10^{-2} \mathrm{~mol} \mathrm{~L}^{-1}\right)$. $\mathrm{O}$ valor de $k_{1}^{\prime}$ para a formação de $\mathrm{CE}\left(2,3 \times 10^{-4} \mathrm{~s}^{-1}\right)$ é da mesma ordem de grandeza, dos valores de $k_{1}(2,5 \pm 0,3) \times 10^{-4} \mathrm{~s}^{-1}$, para o decaimento da concentração de íons cianato em soluções onde as concentrações de etanol são iguais ou inferiores a $8,0 \times 10^{-1} \mathrm{~mol} \mathrm{~L}^{-1}$ (Tabela 1).

Em soluções em que a concentração de etanol é superior a $8,0 \times 10^{-1} \mathrm{~mol} \mathrm{~L}^{-1}$ (ver Tabela 1 ), a constante de velocidade para o decaimento do íon cianato diminui à medida que o teor alcoólico aumenta, atingindo o valor de $5,2 \times 10^{-5} \mathrm{~s}^{-1}$ quando a concentração de $\left[\mathrm{CH}_{3} \mathrm{CH}_{2} \mathrm{OH}\right]$ é de $13,7 \mathrm{~mol} \mathrm{~L}^{-1},\left(80^{\circ} \mathrm{GL}\right)$. A Tabela 2 apresenta os valores dos parâmetros de ativação, para a reação de decaimento de íons cianato, em diferentes concentrações alcoólicas. Coerentemente com as considerações acima, nota-se uma variação de $7 \mathrm{kcal} \mathrm{mol}^{-1}$, $-21 \mathrm{cal} \mathrm{mol}^{-1}$ e $5 \mathrm{kcal} \mathrm{mol}^{-1} \mathrm{~K}^{-1}$ nos valores de $\Delta H_{l}^{+}, \Delta S_{I}^{*}$ e $\Delta G_{I}^{*}$ respectivamente, quando a concentração de etanol varia de $2,0 \times 10^{-2}$ para $6,8 \mathrm{~mol} \mathrm{~L}^{-1}$.

Tabela 2. Parâmetros de ativação para a reação de decaimento de íons cianato em soluções aquo-etanólicas, $\mathrm{pH}=4,5$; temperatura $=25^{\circ} \mathrm{C}(298 \mathrm{~K})$

\begin{tabular}{ccccc}
\hline $\begin{array}{c}{[\mathrm{EtOH}]} \\
\mathrm{mol} \mathrm{L}\end{array}$ & $\begin{array}{c}\Delta H^{\ddagger} \\
\left(\mathrm{kcal} \mathrm{mol}^{-1}\right)\end{array}$ & $\begin{array}{c}\Delta G^{\ddagger} \\
\left(\mathrm{kcal} \mathrm{mol}^{-1}\right)\end{array}$ & $\begin{array}{c}E_{a t}, \\
(\mathrm{kcal} \mathrm{mol})^{2}\end{array}$ & $\begin{array}{c}\Delta S^{\ddagger} \\
\left(\mathrm{cal} \mathrm{mol}^{-1} \mathrm{~K}^{-1}\right)\end{array}$ \\
\hline 6,8 & 20 & 17 & 21 & -10 \\
$2,0 \times 10^{-2}$ & 13 & 22 & 14 & -31 \\
\hline
\end{tabular}

O rendimento químico de carbamato de etila também varia em função da concentração de etanol atingindo os valores de $9,1 \%$ para $\left[\mathrm{CH}_{3} \mathrm{CH}_{2} \mathrm{OH}\right]=8,0 \times 10^{-2} \mathrm{~mol} \mathrm{~L}^{-1}$ e $35 \%$ para $\left[\mathrm{CH}_{3} \mathrm{CH}_{2} \mathrm{OH}\right]$ igual a $10,3 \mathrm{~mol} \mathrm{~L}^{-1} .{ }^{6}$ Estas observações podem ser tentativamente explicadas com base no conjunto de reações simultâneas que podem estar ocorrendo: $:^{20,21}$

$$
\begin{aligned}
& \mathrm{H}^{+}+\mathrm{OCN}^{-} \rightleftharpoons \mathrm{HOCN} \rightleftharpoons \mathrm{HNCO} \\
& \mathrm{HNCO}+\mathrm{H}_{2} \mathrm{O} \rightarrow \mathrm{H}_{2} \mathrm{NCOOH} \\
& \mathrm{H}_{2} \mathrm{NCOOH}+\mathrm{C}_{2} \mathrm{H}_{5} \mathrm{OH} \rightarrow \mathrm{H}_{2} \mathrm{NCOOC}_{2} \mathrm{H}_{5}+\mathrm{H}_{2} \mathrm{O} \\
& \mathrm{NH}_{2} \mathrm{COOH} \rightarrow \mathrm{NH}_{3}+\mathrm{CO}_{2}
\end{aligned}
$$

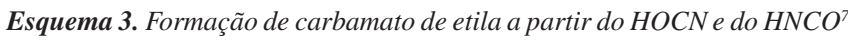

$\mathrm{O}$ ácido ciânico possui uma constante de dissociação de 2,0 x $10^{-4}$ a $28^{\circ} \mathrm{C}$ e apresenta duas formas tautoméricas: o ácido ciânico (HOCN) e o ácido isociânico (HNCO), com predominância do segundo $(97 \%) \cdot{ }^{17,18} \mathrm{O}$ ácido isociânico reagiria com a água originando ácido carbâmico $\left(\mathrm{NH}_{2} \mathrm{COOH}\right)$, o qual pode reagir com etanol originando

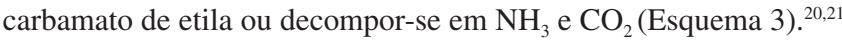
Trata-se de duas reações competitivas, e os rendimentos dos respectivos produtos irão depender das concentrações relativas de água e etanol. Assim, diminuindo a concentração (atividade) da água e aumentando a concentração (atividade) do etanol, a formação de carbamato de etila é favorecida aumentando o seu rendimento. O inverso ocorre com o aumento da concentração (atividade) da água e a diminuição da concentração (atividade) do etanol. Estas considerações, aliadas aos valores numéricos das constantes de decaimento de íons $(\mathrm{OCN})^{-}$na presença e na ausência de etanol, sugerem que o decaimento de íons cianato e não a formação de carbamato de etila, pelo menos nas condições dos experimentos, é a etapa determinante do processo de consumo de $(\mathrm{OCN})^{-}$.

\section{CONCLUSÃO}

Embora mais experimentos sejam necessários para melhor esclarecer o(s) mecanismo(s) da(s) reação(ões) de formação de CE em aguardentes, envolvendo íons cianato, é razoável supor que isto envolve a reação de ácido carbâmico com etanol gerando carbamato de etila. ${ }^{20,21}$

Existe uma diferença substancial entre os parâmetros de ativação $\Delta H_{I}^{*}, \Delta S_{I}^{*}$ e $\Delta G_{I}^{*}$ para a reação de decaimento de íons (OCN)-em função das condições experimentais. Nas soluções onde a concentração de etanol é superior a $8,0 \times 10^{-1} \mathrm{~mol} \mathrm{~L}^{-1}$, a constante de velocidade para esta reação diminui com o aumento da concentração de etanol. O inverso é observado quando ocorre o aumento da concentração de água. As alterações nas concentrações da água e do etanol afetariam as constantes de velocidade de formação de carbamato de etila e de decomposição do íon $(\mathrm{OCN})^{-}$, favorecendo de forma diversa estas reações competitivas. Este tema esta sendo investigado em nosso Laboratório.

\section{AGRADECIMENTOS}

CNPq, Capes e FAPESP pelo auxílio financeiro.

\section{REFERÊNCIAS}

1. Madrera, R. R.; Valles, B. S.; Food Control 2009, 20, 139.

2. Zimmerli, B.; Schlatter, J.; Mutat. Res. 1991, 259, 325

3. Weber, J. V.; Sharypov, V. I.; Environ. Chem. Lett. 2009, 7, 233.

4. Lachenmeier, D. W.; Lima, M. C. P.; Nóbrega, I. C. C.; Pereira, J. A. P.; Kerr-Corrêa, F.; Kanteres, F.; Rehm, J.; BMC Cancer 2010, 10, 1.

5. Dongpeng, W. Yang, B.; Zhai, L. Z.; Fuel Process. Technol. 2007, 88, 807.

6. Galinaro, C. A.; Tese de Doutorado, Universidade de São Paulo, Brasil, 2011.

7. Boulton, R.; Em Elaboration et connaissance des spiritueux; Cantagrel, R., ed.; Lavoisier-Tec \& Doc: Paris, 1992. cap. 4.

8. Ough, C. S.; Crowell, E. A.; Gutlove, B. R.; Am. J. Enol. Vitic. 1988, 39, 239.

9. Ough, C. S.; Crowell, E. A.; Mooney, L. A.; Am. J. Enol. Vitic. 1988, 39, 243.

10. Alexandrova, A. N.; Jorgensen, W. L.; J. Phys. Chem. B 2007, 111, 720.

11. Aresta, M.; Boscolo, M.; Franco, D. W.; J. Agric. Food Chem. 2001, 49, 2819.

12. Guilloton, M.; Karst, F. A.; Anal. Biochem. 1985, 149, 291.

13. Clegg, B. S.; Frank, R. J. Agric. Food Chem. 1988, 36, 502.

14. Lachenmeier, D. W.; Frank, W.; Kuballa, T.; Rapid Commun. Mass Spectrom. 2005, 19, 108 .

15. Andrade-Sobrinho, L. G.; Boscolo, M.; Lima-Neto, B. S.; Franco, D. W.; Quim. Nova 2002, 25, 1074.

16. Andrade-Sobrinho, L. G.; Capellini, L. T. D.; Da Silva, A. A.; Galinaro, C. A.; Buchviser, S. F.; Cardoso, D. R.; Franco, D. W.; Quim. Nova 2009, 32, 116 .

17. Well, A. F.; Structural Inorganic Chemistry, $5^{\text {th }}$ ed., Oxford University Press: London, 1975.

18. Vrieze, K.; Van Koten, G. Em Comprehensive Coordination Chemistry; Wilkison, G.; Gillard, R. D.; McClaverty, J. A., eds.; Pergamon: Oxford, 1987, v. 2, cap. 13.5 .

19. Bunnett, J. F. Em Investigation of Rates and Mechanisms of Reaction Part I, Lewis, E. S., ed.; Wiley: New York, 1974, v. 6, cap. 8.

20. Amell, A. R.; J. Am. Chem. Soc. 1956, 78, 6234.

21. Adams, P.; Baron, F. A.; Chem. Rev. 1965, 65, 567. 